\title{
Appendagite aiguë
}

\section{Primary epiploic appendagitis}

\section{Pereira $\cdot$ S. Laribi $\cdot$ L. Hamzi $\cdot$ P. Plaisance}

Reçu le 17 février 2012 ; accepté le 30 mai 2012

(C) SFMU et Springer-Verlag France 2012

Un homme de 44 ans consulte pour une vive douleur en fosse iliaque gauche (FIG). L'examen clinique abdominal ne retrouve qu'une défense en FIG sans symptôme digestif associé ni syndrome inflammatoire biologique. Devant ce tableau clinique, une tomodensitométrie est pratiquée. Sur cette coupe axiale de scanner abdominal avec opacification colique et injection en intraveineuse (IV) de produit de contraste iodé, on observe une image de forme ovalaire spontanément hypodense, de densité graisseuse, bien limitée par un fin liseré hyperdense. Cette image est discrètement hétérogène, extracolique, sous séreuse, associée à une infiltration de la graisse en regard de la face antérieure du côlon gauche dont l'aspect est typique d'une torsion de frange épiploïque aussi appelée appendagite aiguë (Fig. 1). Ces éléments d'imagerie sont caractéristiques et très souvent rehaussés après injection IV de produit de contraste. Dans la grande majorité des cas, ils sont situés sur le colon gauche et plus rarement au niveau caecal. Cette image scannographique doit être connue du médecin urgentiste. La reconnaissance d'une appendagite aiguë peut permettre d'éviter une intervention chirurgicale et/ou une antibiothérapie inutile. En effet, le traitement est ambulatoire par antalgiques, certains préconisant d'y associer des anti-inflammatoires non stéroïdiens [1].

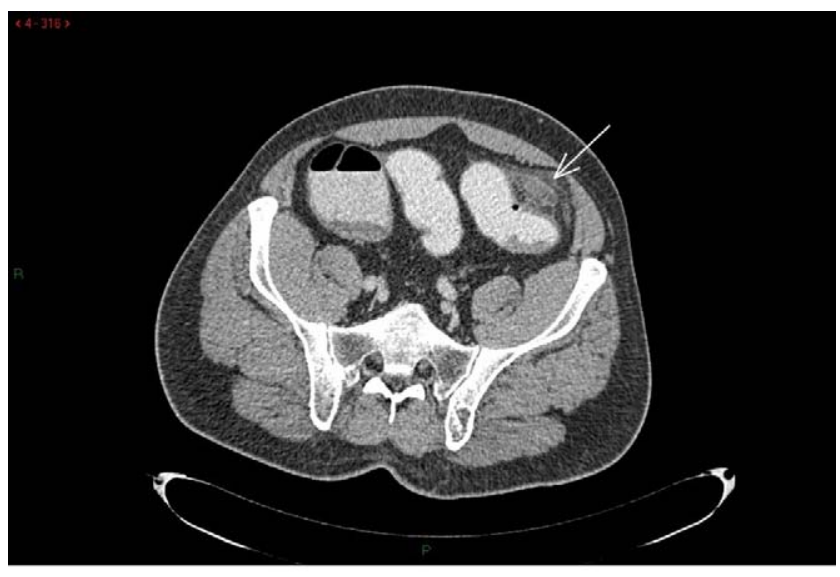

Fig. 1 Image de forme ovalaire spontanément hypodense, de densité graisseuse, discrètement hétérogène, extracolique, sous séreuse, associée à une infiltration de la graisse en regard de la face antérieure du côlon gauche dont l'aspect est typique d'une torsion de frange épiploïque aussi appelée appendagite aiguë

\section{Référence}

1. Vinson DR (1999) Epiploic appendagitis: a new diagnosis for the emergency physician. Two case reports and a review. J Emerg Med 17:827-32

M. Pereira $\cdot$ S. Laribi $(\varangle) \cdot$ P. Plaisance

Service d'accueil des urgences,

groupe hospitalier Saint-Louis-Lariboisière, APHP,

2, rue Ambroise Paré, F-75010 Paris, France

e-mail : said.laribi@lrb.aphp.fr

\section{Hamzi}

Service de radiologie,

groupe hospitalier Saint-Louis-Lariboisière, APHP,

2, rue Ambroise Paré, F-75010 Paris, France

P. Plaisance

Université Paris Diderot-Paris 7, 5, rue Thomas-Mann,

F-75205 Paris cedex 13, France 